\title{
The Effects of Low Dose-Rate Ionizing Radiation on the Shapes of Transients in the LM124 Operational Amplifier
}

\author{
Stephen Buchner, Member, IEEE, Dale McMorrow, Member, IEEE, Nicholas Roche, Student \\ Member, IEEE, Laurent Dusseau, Senior Member, IEEE, and Ron L. Pease, Fellow, IEEE.
}

\begin{abstract}
Shapes of single event transients (SETs) in a linear bipolar circuit (LM124) change with exposure to total ionizing dose (TID) radiation. SETs shape changes are a direct consequence of TID-induced degradation of bipolar transistor gain. A reduction in transistor gain causes a reduction in the drive current of the current sources in the circuit, and it is the lower drive current that most affects the shapes of large amplitude SETs.
\end{abstract}

\section{INTRODUCTION}

$\mathrm{S}$ ingle event transients (SETs) appear in linear bipolar circuits exposed to heavy ions, such as are present in the space radiation environment. Such circuits are expected to operate for a number of years, during which time they accumulate an ionizing radiation dose that could, if the circuits are not radiation-hardened, affect their electrical properties. Radiation-induced changes in a circuit's electrical characteristics will modify the shapes of SETs. This is of concern to the system designer, because such changes could increase, or decrease, system error rates. Therefore, it is necessary to obtain a better understanding of how TID affects SETs.

The first demonstration that TID does, in fact, alter the shapes of SETs in linear bipolar circuits involved the LM139 voltage comparator [1]. SETs were generated by focusing pulsed laser light onto sensitive circuit nodes, consisting of bipolar transistors, and monitoring the output with an oscilloscope. When the input voltage on $\mathrm{V}_{\text {in }}(+)$ was greater than the input voltage on $\mathrm{V}_{\text {in }}(-)$, all SETs had negative amplitudes, and exposure to TID caused a reduction in the slopes of the leading edges of the SETs. The reduced slopes

Manuscript received July 11th, 2008. This work was supported in part by NASA Electronic Parts and Packaging Program and by Defense Threat Reduction Agency.

Stephen Buchner is with QSS/PSGS, Seabrook, MD 20706 USA (phone: 301-286-5019; fax: 301-286-4699; e-mail: stephen.p.buchner@ nasa.gov).

Dale McMorrow is with the Naval Research Laboratory, Washington, DC, 20375, USA. (e-mail: mcmorrow@ccs.nrl.navy.mil).

Nicholas Roche is with Universitè Montpellier II, F-34095 Montpellier cedex 5, France (e-mail : roche@ies.univ-montp2.fr).

Laurent Dusseau is with Universitè Montpellier II, F-34095 Montpellier cedex 5, France (e-mail : dusseau @ ies.univ-montp2.fr).

Ron L. Pease is with RLP Research, Albuquerque, NM, 87031 USA (email: 1srlpease@ wildblue.net). resulted in smaller SET amplitudes that translate into lower system error rates with increasing total dose.

A subsequent study described the results of pulsed-laser and heavy-ion experiments on the LM124 operational amplifier [2]. The shapes of the SETs at the output of the LM124 are very different from those of the LM139, i.e., some SETs are unipolar with either positive or negative amplitudes, whereas others are bipolar with different amounts of positive and negative contributions. The study compared SETs generated at three different locations in a pristine LM124 circuit with those in a circuit exposed to a total ionizing dose (TID) of 150 $\operatorname{krad}(\mathrm{Si})$ at a dose rate of about $1 \operatorname{rad}(\mathrm{Si}) / \mathrm{s}$. The changes in SET shapes were also compared for three different amplifier configurations, i.e., voltage follower (VF), inverter (INV) with a gain of 10, and non-inverter (NINV) with a gain of 11. SETs in irradiated devices had noticeably different shapes from those in pristine devices, i.e., the transients at some locations had reduced amplitudes following irradiation, whereas others showed only small changes in amplitude but large increases in width.

The results reported above were for only two transistors and one resistor in a device irradiated to a dose of $150 \mathrm{krad}(\mathrm{Si})$. Because no electrical measurements were done, it was not possible to relate the changes in SET shapes to changes in electrical parameters. The present study differs from the previous one in that electrical parameters and SET shapes were measured as a function of TID. The electrical parameters included open loop gain, input current, input offset voltage, power supply current, and slew rate. The electrical measurements had two purposes: one was to determine when the part exceeded the manufacturer's specifications, and the other was to relate changes in electrical parameters to changes in SET shapes. Along with the electrical measurements, SET shapes were captured for thirteen different nodes in the LM124.

\section{TEST SETUP AND EXPERIMENTAL DESCRIPTION}

The LM124 consists of four operational amplifiers on a single chip. Fig. 1 is a photomicrograph of one of the four amplifiers, with the transistors that were probed with the laser beam clearly marked. Fig. 2 is a circuit diagram of the LM124. 


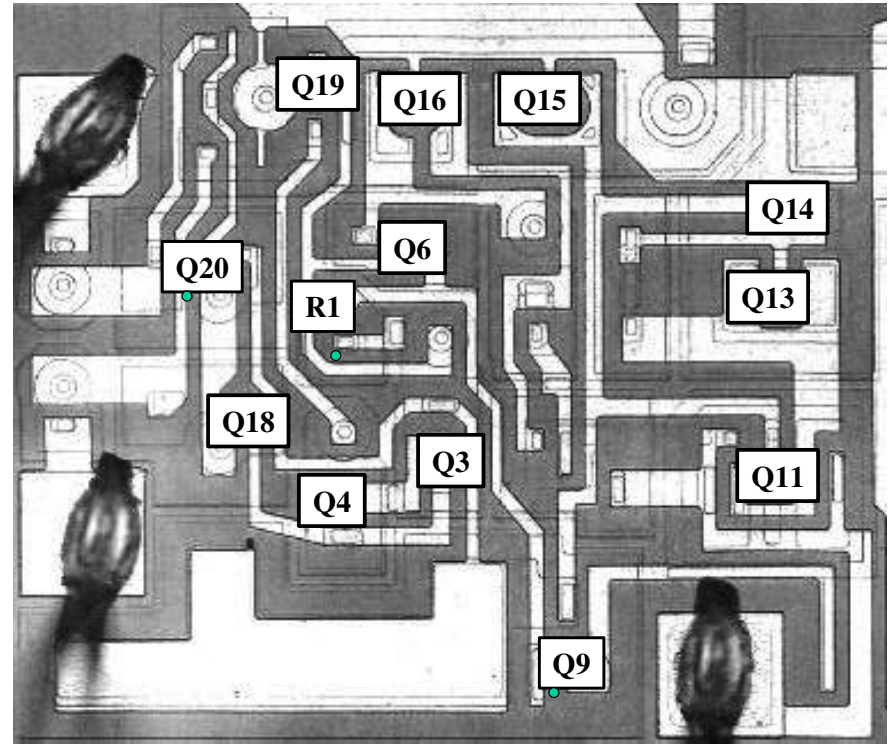

Fig. 1. Photomicrograph of one of the four amplifiers on the LM124 chip. The locations of the twelve transistors and one resistor probed with the pulsed laser are indicated.

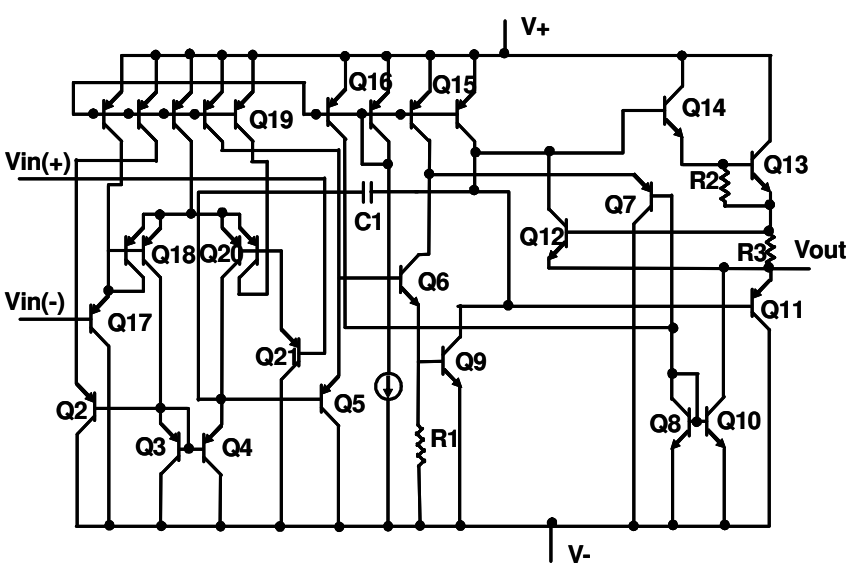

Fig. 2. Circuit diagram for the LM124, showing the transistors and resistor that were probed with the laser.

To measure SET dependence on amplifier configuration and TID, three of the four operational amplifiers were electrically connected, one as a VF, another as an INV (gain of 10) and a third as a NINV (gain of 11). The fourth op-amp was not connected.

One part was de-lidded and exposed to ionizing radiation in the NASA-GSFC ${ }^{60} \mathrm{Co}$ gamma cell at a dose rate of 10 $\operatorname{mrad}(\mathrm{Si}) / \mathrm{s}$. During irradiation, the part was held at room temperature and voltages of $+5 \mathrm{~V}$ and $-5 \mathrm{~V}$ were applied to $\mathrm{V}_{\mathrm{dd}}$ and $\mathrm{V}_{\mathrm{ss}}$, respectively. All inputs were grounded and the outputs were left unconnected (floating). After receiving an incremental ionizing dose, the part was removed from the chamber and the electrical parameters measured before being transported to the pulsed laser test facility at the Naval Research Laboratory. (See [3] for experimental details.) The pristine and irradiated parts were mounted, end-on-end, in a single socket to facilitate the direct comparison of SETs in the two devices. A bias of $0.13 \mathrm{~V}$ was applied to all three opamps, resulting in outputs of $0.13 \mathrm{~V}$ for the $\mathrm{VF},-1.3 \mathrm{~V}$ for the INV and $1.43 \mathrm{~V}$ for the NINV. A low-capacitance $(11 \mathrm{pF})$ oscilloscope probe was connected to the outputs. The energy of each laser pulse was measured by diverting a small portion of the beam onto a calibrated photodiode.

The first step was to ensure that the experimental setup for the pulsed laser did not change from one set of measurements to the next by positioning the pristine device in front of the focusing lens and adjusting, if necessary, the optical path for the laser light through the focusing optics until all the SETs in the pristine device were the same as those measured and captured during the previous run. Next, the irradiated device was moved into the focused laser beam and transients from twelve transistors and one resistor were collected and stored for the VF configuration. This procedure was repeated for the other two amplifier configurations.

The choice of relatively large laser pulse energies was dictated by a number of factors. First was the desire to follow the declining SET amplitudes with dose up to $50 \mathrm{krad}(\mathrm{Si})$. Second was the realization that, for use in a system deployed in a radiation environment, it is most important to protect against the largest SETs. Therefore, large energies that would generate large SETs with amplitudes close to saturation were selected. For transistor Q9 (one of the most sensitive transistors), the energy was $4.5 \mathrm{pJ}$ (equivalent LET $=13.5$ $\mathrm{MeV} . \mathrm{cm}^{2} / \mathrm{mg}$ ). For transistors Q3, Q4 and Q18, the energy was $15 \mathrm{pJ}$ (equivalent LET $=45 \mathrm{MeV} \cdot \mathrm{cm}^{2} / \mathrm{mg}$ ), and for the remaining transistors, the energy was $34 \mathrm{pJ}$ (equivalent LET $=$ $100 \mathrm{MeV} . \mathrm{cm}^{2} / \mathrm{mg}$ ).

The electrical and pulsed-laser tests were completed in one day, after which the part was returned to the ${ }^{60} \mathrm{Co}$ gamma cell for the next irradiation step. Given the low dose rate, no annealing is expected during the time the part was undergoing testing.

\section{RESULTS}

Due to space limitations, it is not possible to include in this paper all the data for the twelve transistors and one resistor in the three different amplifier configurations. Instead, data for just a few transistors, that are emblematic of the entire set, will be presented. The data will illustrate two important points: i) the shapes of the SETs are determined by the electrical configuration of the amplifiers, and ii) the electrical measurements of slew rate and open loop gain can explain the TID-induced changes in shapes.

\section{A. Transistor $Q 9$.}

Figs. 3, 4, and 5 present the SETs generated at transistor Q9 for three different amplifier configurations. The laser light was focused on the base of Q9, which is the most sensitive region. Fig. 3 shows that, in the VF configuration, the initial SET has a negative amplitude that is just at saturation $(-5 \mathrm{~V})$ and the trailing edge exhibits a straight-line recovery over a period of about $25 \mu \mathrm{s}$. With increasing dose, both the amplitude and the slope of the trailing edge decrease. 


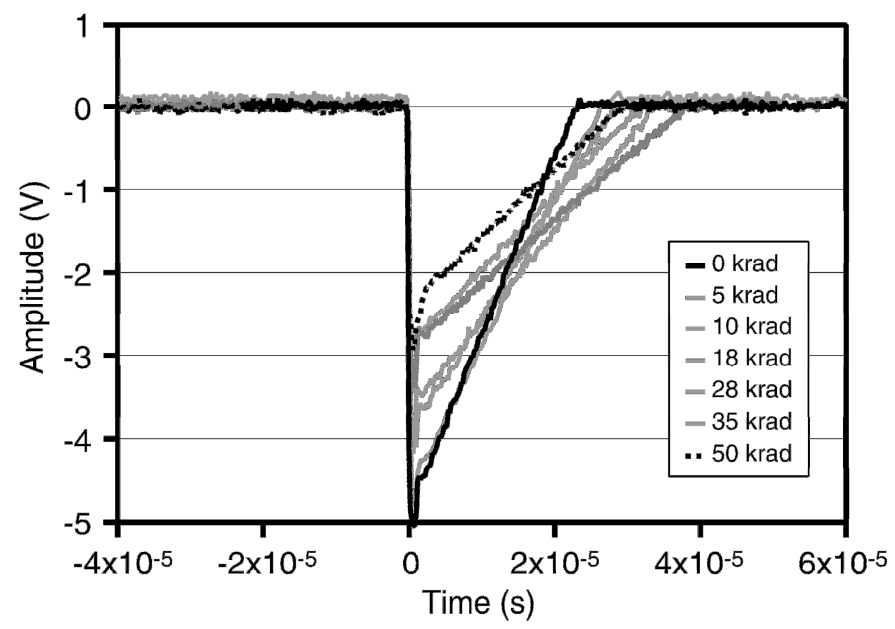

Fig. 3. SETs for transistor Q9 in the VF configuration as a function of TID. With increasing dose, the amplitude decreases and the slope increases.

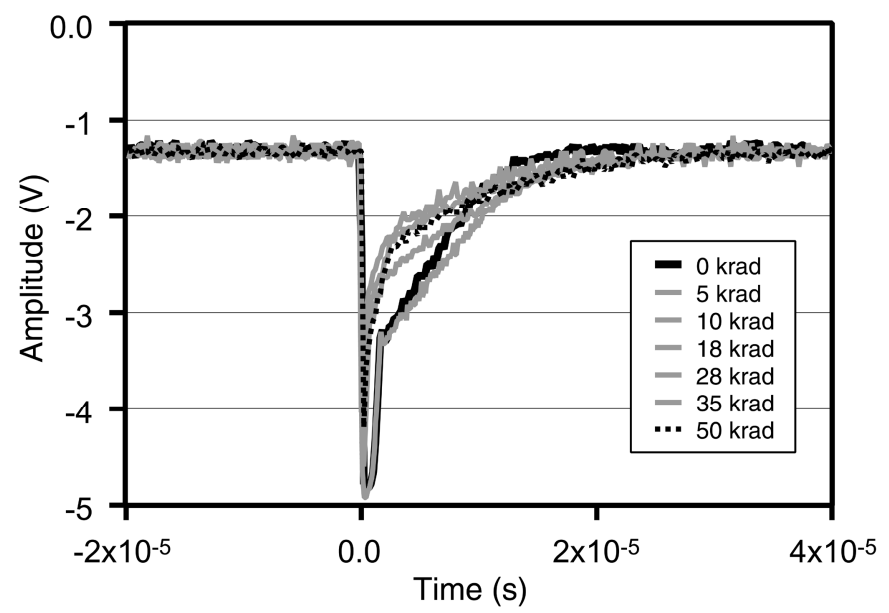

Fig. 4. SETs for transistor Q9 in the INV configuration as a function of TID. With increasing dose, the amplitude decreases and the pulse width increases.

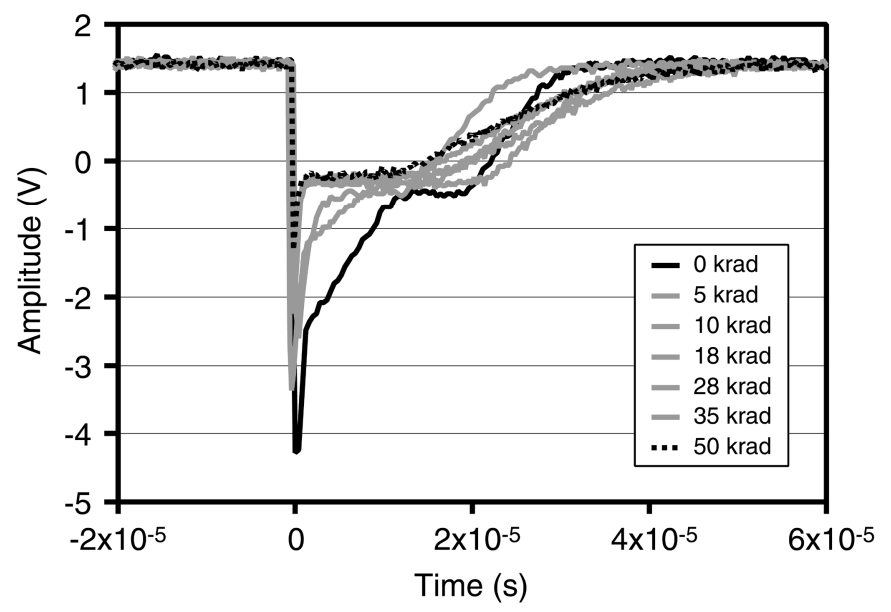

Fig. 5. SETs for transistor Q9 for the NINV configuration as a function of TID. With increasing dose, the amplitude decreases.

Fig. 4 shows that, for the INV configuration, the SET has a different shape. Although the amplitude is also negative and decreases with TID, the SET is characterized by a narrow negative peak and a compound trailing edge in which the initial recovery is a straight line and the final recovery is an exponential curve that has an RC time constant of approximately $10 \mu \mathrm{s}$. With increasing dose the straight line contribution decreases and more of the recovery consists of an exponential dependence on time.

Fig. 5 shows that SETs in the NINV configuration have characteristics in common with those observed in the VF and INV cases, such as negative amplitudes, narrow negative peaks, and amplitudes that decrease with TID. However, there is an additional unique feature in the form of a plateau in the trailing edge of the SET. The duration of the plateau, and the voltage at which it occurs, also depend on TID. Following the plateau, the trailing edge also exhibits a recovery similar to that of the INV case where initially it is linear with time and then becomes exponential.

\section{B. Transistor Q6}

SETs generated at Q6 are very different from those generated at Q9. Fig. 6 shows SETs generated at Q6 for the VF configuration with the light focused on the base region. The SET is bipolar with a dominant initial positive component and a much smaller negative component that has a straight-line recovery over a time interval of approximately $50 \mu \mathrm{s}$. With increasing dose, the positive component becomes smaller and narrower, and the negative component disappears.

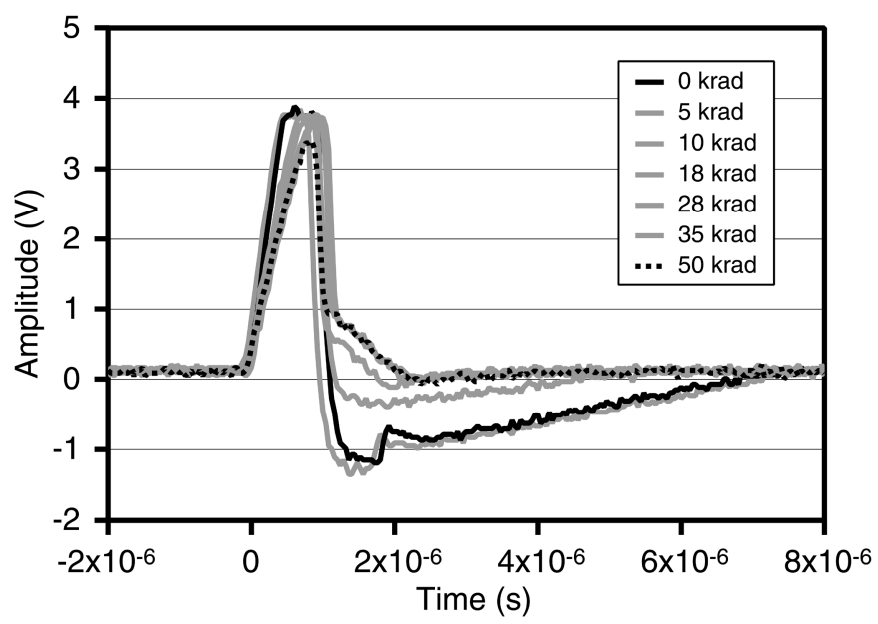

Fig. 6. SETs generated at transistor Q6 in the VF configuration as a function of TID. With increasing dose, the negative component gradually disappears. 


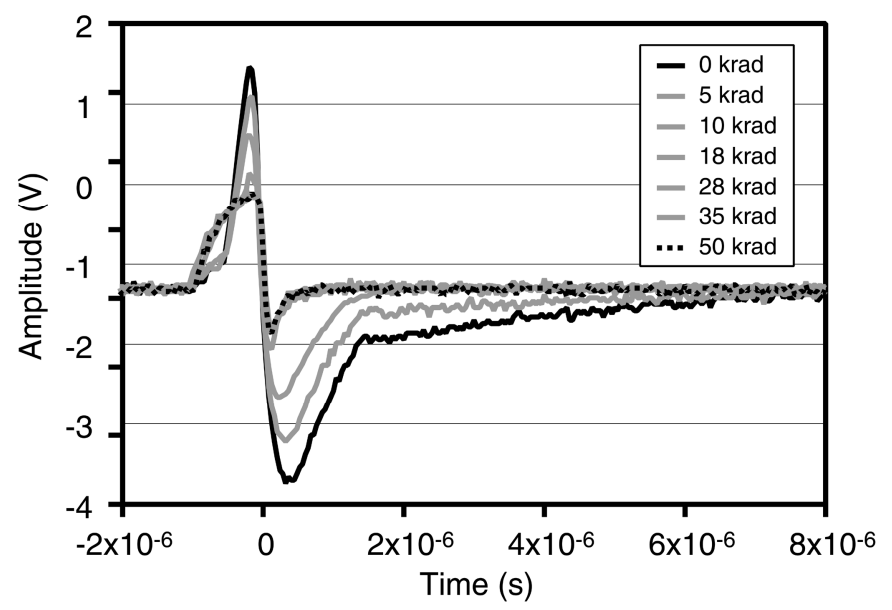

Fig. 7. SETs generated at transistor Q6 for the INV configuration as a function of TID. With increasing dose, both the positive and negative peaks decrease in amplitude.

Fig. 7 shows the SETs for the INV configuration. The initial SET is markedly bipolar in character, with positive and negative components of comparable size. Both components decrease with increasing TID.

Fig. 8 shows the SETs generated at Q6 in the NINV case. They are similar to those for the VF, except that their amplitudes are predominantly positive with a very small negative component. With increasing dose, the positive component barely changes, while the negative component almost disappears. Note the absence of plateaus in the data for the NINV.

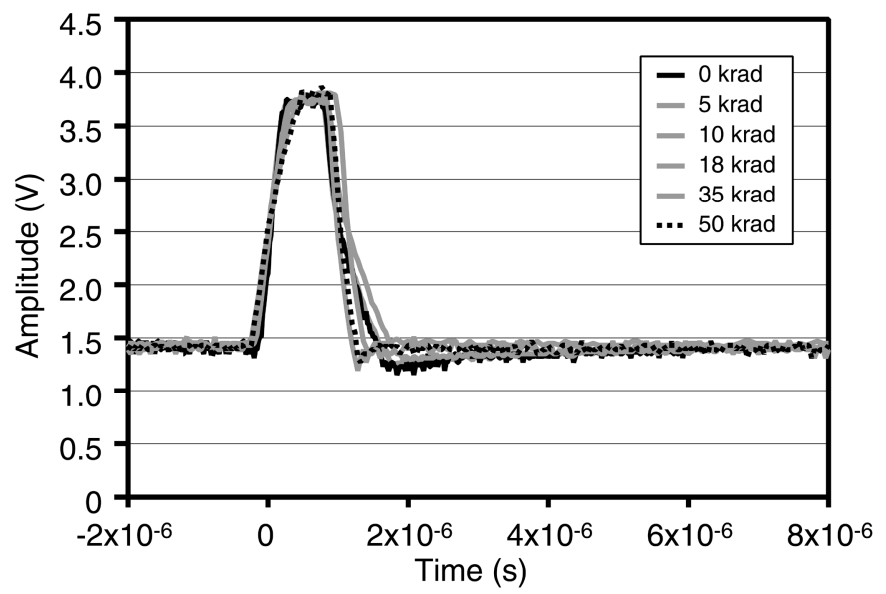

Fig. 8. SETs generated at transistor Q6 for the NINV configuration as a function of TID. With increasing dose, the negative component disappears.

\section{Transistor $Q 4$}

The SET data for transistor Q4 are included to illustrate the wide variety of SET shapes that originate in the LM124. Fig. 9 shows the SETs for transistor Q4 in the VF configuration. The SETs are unipolar with positive amplitude and a straightline recovery, whose slope decreases with increasing TID. The linear recovery, previously observed for SETs in the VF configuration for transistor Q9, is also observed for SETs originating in a number of other transistors, including Q13,
Q14, Q15, Q16, Q18, Q19, and Q20. Note that the amplitude decreases by about $15 \%$ after a TID of $50 \mathrm{krads}(\mathrm{Si})$.

Fig. 10 shows the data for transistor Q4 in the INV configuration. The SETs also have positive amplitudes but the trailing edges have an exponential recovery with an RC time constant of about $8 \mu \mathrm{s}$. For this case, the amplitude appears to increase slightly with TID rather than to decrease, as seen in most of the other SETs.

Fig. 11 shows the data for transistor Q4 in the NINV configuration. The SETs are similar to those in the INV configuration in that they have positive amplitudes and exponential recoveries, but the amplitudes appear to be independent of TID. With increasing dose, the trailing edges take longer to recover and, by fitting the data with exponentially decaying curves, the recovery times are seen to increase by a factor of three following exposure to a TID of 50 $\operatorname{krad}(\mathrm{Si})-$ from $3.1 \mu \mathrm{s}$ to $9.5 \mu \mathrm{s}$.

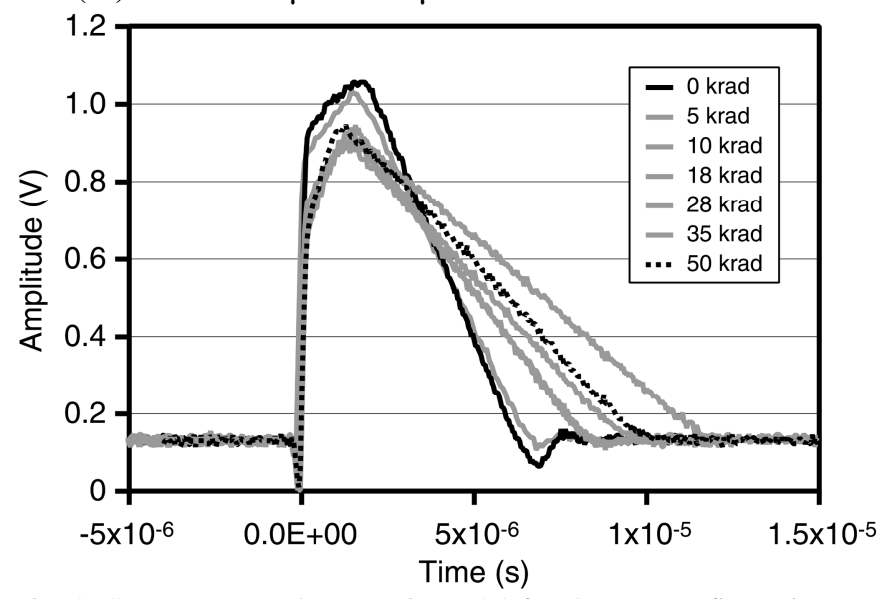

Fig. 9, SETs generated at transistor Q4 for the VF configuration as a function of TID. With increasing dose, both the amplitude and the trailing-edge slope decrease.

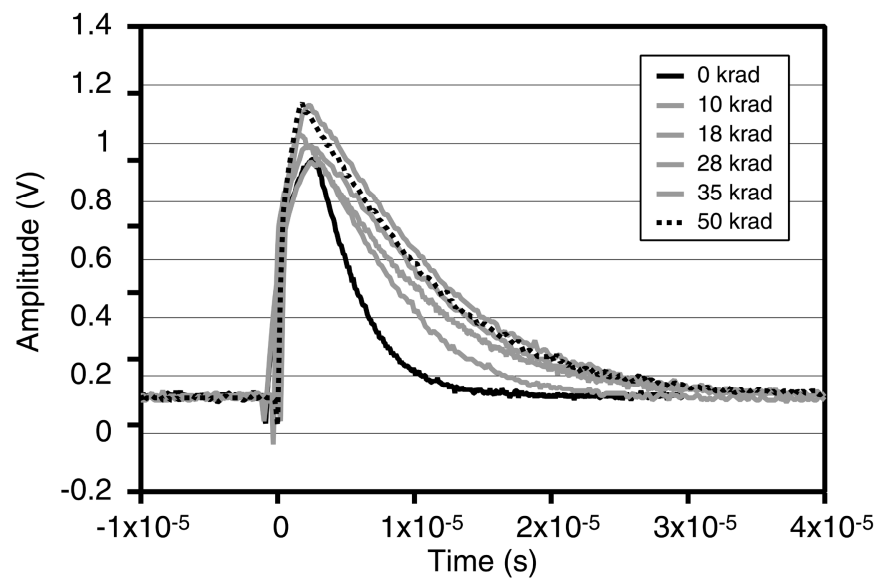

Fig. 10. SETs generated at transistor Q4 for the INV configuration as a function of TID. With increasing dose, the amplitude and width increase. 


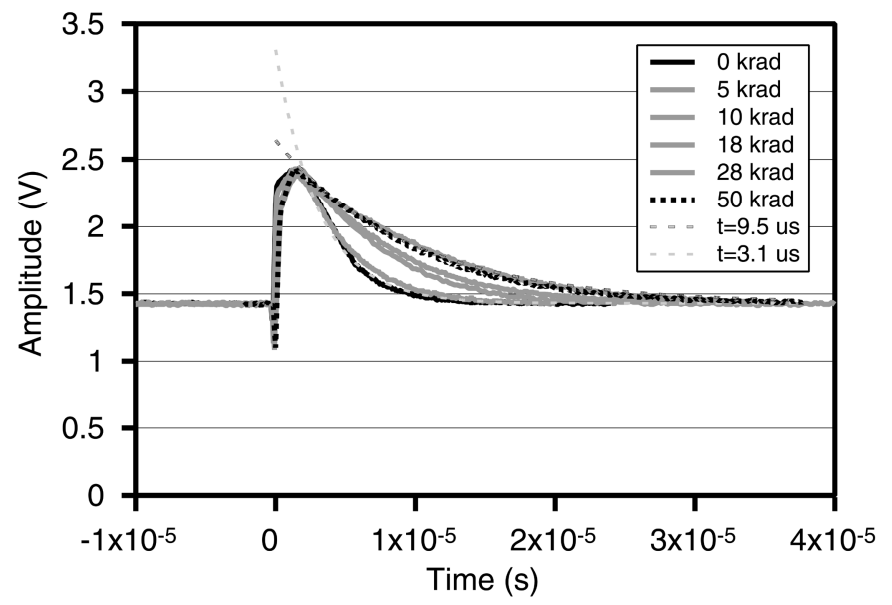

Fig. 11. SETs generated at transistor Q4 for NINV configuration as a function of TID. Exponential decay times were obtained by fitting to the trailing edges of SETs for $0 \mathrm{krad}(\mathrm{Si})$ and $50 \mathrm{krad}(\mathrm{Si})$. With increasing dose, the width of the transient increases.

\section{Electrical Measurements}

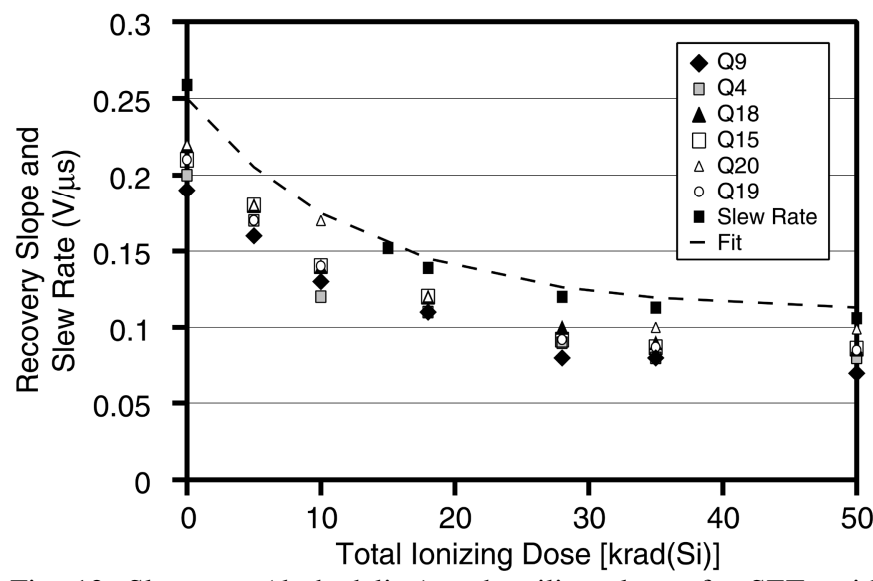

Fig. 12. Slew rate (dashed line) and trailing slopes for SETs with straight line recoveries in the VF configuration.

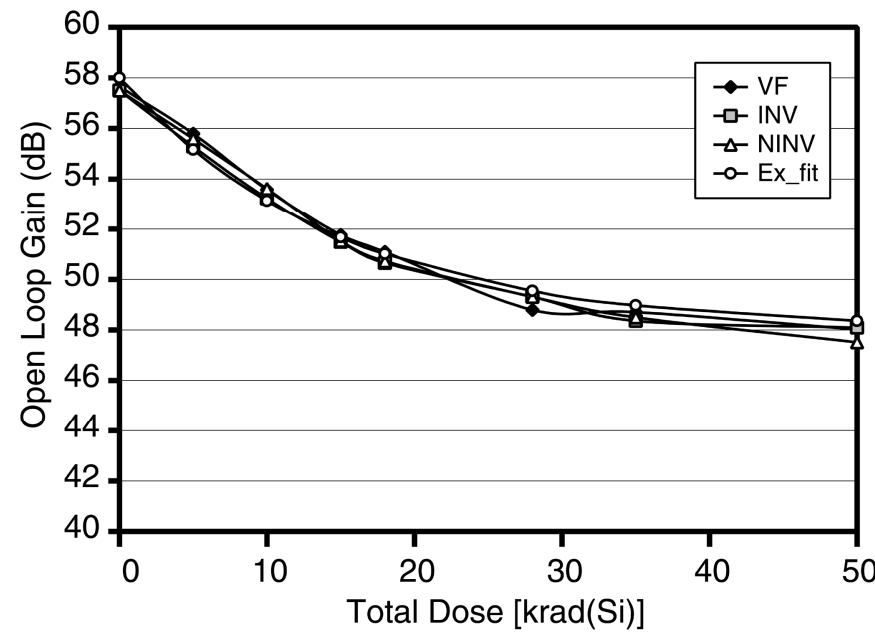

Fig. 13. Open loop gain at $1 \mathrm{KHz}$ as a function of TID for the three op-amps tested in the LM124. The minimum specified in the data sheet is $28 \mathrm{~dB}$. The experimental fit is a least squares fit using an exponential decay function [Gain $=10 \bullet \exp (-\mathrm{TID} / 14)+48$ ].
As already mentioned, electrical parameters were measured after each incremental radiation dose. A parametric analyzer (HP4156B) was used for some of the measurements and a signal generator and oscilloscope were used for measuring slew rate. Fig. 12 shows a comparison of the slew rate measured for the device in the VF configuration with the slopes of the trailing edges of a number of SETs originating from different transistors also in the VF configuration. The decrease in the slew rate with TID tracks the decreases in slope of the trailing edges of the SETs.

The open loop gain, measured at $1 \mathrm{KHz}$, also decreases with TID and has the same dependence on TID as the slew rate.

\section{DISCUSSION}

The data presented above clearly indicate that the SETs generated in the LM124 operational amplifier depend on a number of different factors, including the origins of the SETs in the LM124 circuit itself, i.e., which transistor is struck by the incident heavy ion, the amount of energy deposited to trigger the SET, and the electrical configuration of the amplifier.

To explain qualitatively the shapes of the SETs requires a detailed understanding of how each transistor in the entire circuit responds to charge injected at just one node. The explanation can be quite complicated, because the ultimate shapes of the SETs at the outputs are determined not only by the properties of the transistors where the charges are injected, but by all the transistors that "sense" the changes in current or voltage as the SETs propagate from source to output.

A quantitative explanation requires modeling the circuit's response to charge injected at specific locations in the circuit using a program such as SPICE [4]. Detailed information is required on how each transistor degrades with TID. The degradation of individual transistors depends on their type (npn or pnp), physical layout and bias configuration, which are different for each transistor. So there is no way to know, a priori, how much the degradation of each transistor will contribute to the overall degradation of the circuit.

We have opted instead for a qualitative approach, because it provides greater insight into the mechanisms responsible for the SET shapes. Still, because the explanations can be rather compicated, we will discuss only two transistors in detail. The two selected, Q9 and Q6, have relatively low energy thresholds for producing SETs. Our qualitative approach was simplified by the fact that we chose to consider large-signal SETs for which we could relate the measured electrical parameters of slew rate and open loop gain to the slopes of the trailing edges of some of the SETs.

\section{A. Transients at $Q 9$ in the VF Configuration}

The signs of the SETs originating at transistor Q9 may be determined by the following analysis. Injecting charge into the base of Q9 lowers the voltage on the base of Q11. In the VF configuration, Q11 reproduces the voltage drop, lowering the output voltage. The resulting SET should have negative amplitude, regardless of the amplifier configuration, i.e. VF, 
INV, or NINV.

The large amount of charge injected into the base of Q9 drives the SET to the rail at $-5.0 \mathrm{~V}$. The SET has a relatively narrow negative peak that is less than $1 \mu$ s wide and is the direct response of the circuit to the injected charge. The sharp negative peak is followed by a linear rise in the voltage until it reaches the DC level of $0.13 \mathrm{~V}$.

In the VF configuration, the output is connected directly to the inverting input terminal, which has very high input impedance and no current flows. The output, which consists of a Darlington pair (Q14 and Q13) together with Q11, is biased with a $50 \mathrm{~mA}$ current source consisting of transistors Q8 and Q10. The Darlington pair is in a common collector (emitter-follower) configuration, which is a "Class A" output stage in which the output always follows the input [5].

The output signal ( $-5 \mathrm{~V}$ amplitude) fed back to the input is considerably larger than the voltage at which the input transistors are saturated $(0.12 \mathrm{~V})$. When the input transistors are in saturation, no signal can pass through and the output does not follow the input. The analysis involves using the large-signal approximation, similar to what is used for explaining the slew rate measurements. The recovery characteristics reflect the recharging of capacitor $\mathrm{C} 1$ by the current sources. With increasing dose the drive current is degraded and results in longer times needed to recharge $\mathrm{C} 1$. The longer recharging times lead to SET recoveries with smaller slopes.

\section{B. Transients at $Q 9$ in the INV Configuration}

The SETs originating in transistor Q9 in the INV configuration also have a narrow negative peak with a width of about $1.5 \mu \mathrm{s}$. In the inverting configuration, a current can flow between the input and the output, depending on the sign of the SET. The output is now designated as a "Class AB" output stage that consists of two transistors (Q13 and Q11) that act as a "push-pull" pair. The recovery starts out as a straight line because, as in the case of the VF, the input transistors are driven into saturation by the large feedback signal. However, because the feedback signal is attenuated by a factor of 10 due to the feedback resistor, when the output signal's amplitude drops below $1.2 \mathrm{~V}$, the signal at the inverting terminal drops below $120 \mathrm{mV}$, and the input transistors are no longer saturated. The output responds to the input and the amplifier returns to the linear response regime. As a result, the decay of the SET switches from straight line to exponential until it reaches its DC level of -1.3 Volts. Fig. 4 clearly shows that the recovery switches from linear to exponential when the SET drops below approximately $-3.1 \mathrm{~V}$.

\section{Transients at $Q 9$ in the NINV Configuration}

For the NINV configuration, the SETs again have narrow negative peaks followed by straight-line recoveries. However, unlike the case of the INV, the recoveries are interrupted by plateaus that last for approximately $10 \mu \mathrm{s}$. After the plateau, the recoveries continue on a straight line until they cross close to $0 \mathrm{~V}$, where they switch to an exponential decay.
As for the case of the INV, the straight-line recovery for the NINV is due to the fact that the output signal is greater than $1.2 \mathrm{~V}$, which puts the system into the large-signal regime. The system only leaves the large-signal regime when the negative amplitude is less than $1.2 \mathrm{~V}$, which, because the DC level is at $1.43 \mathrm{~V}$, means the transformation occurs near $0 \mathrm{~V}$. The plateau appears because of the push-pull output stage. For positive signals, Q13 is on and Q11 is off. For negative signals, the opposite is the case. However, the transistors do not switch at the same input voltage, which means that there is a voltage span around $0 \mathrm{~V}$ where both Q11 and Q13 are off, effectively isolating the output and preventing it from changing. The result is a plateau.

It should be stressed that the plateau is only present when the SET signal crosses zero voltage for the INV and NINV configurations. A simple experiment was performed to confirm this. Fig. 14 presents a series of SETs for different laser pulse energies. The figure clearly shows that the plateau is present as long as the SET has negative amplitude that puts it below $0.6 \mathrm{~V}$. When the amplitude is smaller, the plateau disappears.

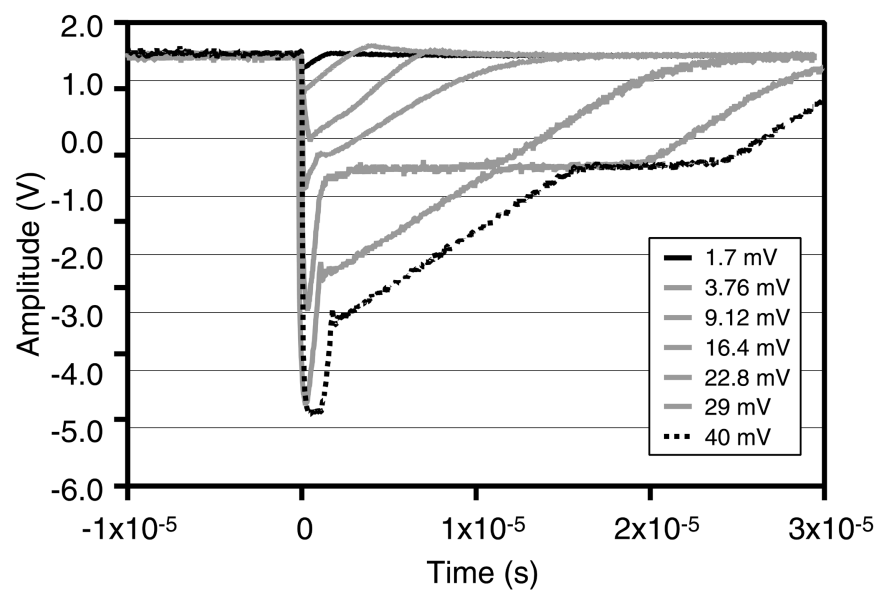

Fig. 14. SETs in a pristine device captured at Q9 over a range of laser pulse energies. At sufficiently small energies, the negative amplitude does not reach $-0.6 \mathrm{~V}$, and the plateau disappears. The units in the legend are the readings (in $\mathrm{mV}$ ) on the photodiode used to measure the laser pulse energy.

The plateau is absent in the INV case because the DC level is at a negative voltage and the SET is also negative, so the output never comes close to the region where the two pushpull transistors are both off. This would also apply for positive SETs for the NINV case, as is seen in the data for transistor Q4 (Fig.11).

\section{Transients at Q6 in the VF Configuration}

Injecting charge into the base of Q6 lowers the base voltage from $1.2 \mathrm{~V}$ to $0.6 \mathrm{~V}$. This in turn lowers the emitter voltage of Q5 and, because of the diode between the emitter and base of Q5, the base voltage is also lowered. Since Q6 is biased, it recovers rapidly while the compensation capacitor maintains the voltage drop at the base of Q5 much longer. This injects a negative signal at the input of the common emitter stage that is amplified and inverted to produce a positive SET at the output. 
The positive signal is fed back to the inverting input terminal, and because it is an inverting terminal, it will be negative at the output. The output signal is the combination of the positive signal from Q6 and the negative signal re-injected by the feedback loop. In the VF configuration, the two inputs are at $0.13 \mathrm{~V}$. The positive component of the SET is entirely reinjected at the negative input terminal. This feedback signal is much larger than in the NINV and produces a bipolar signal. The negative signal saturates the differential pair that recovers slowly, exhibiting a slope limited by the slew rate.

\section{E. Transients at Q6 in the INV Configuration}

The SETs originating at transistor Q6 in the INV configuration are bipolar, with comparable positive and negative components. Note that both the positive and negative amplitudes decrease with increasing dose.

In the case of the VF (described above) and the NINV (described below) the baseline of the output signal is positive. This implies that Q9 has a reduced conduction and Q5 is on. Therefore, charge injected into the base of Q6 will increase the conduction of Q5 and reduce the conduction of Q9 but will not change the state of the transistors, which favors the positive component of the SET.

In the INV configuration, the baseline is at $-1.3 \mathrm{~V}$, which means that Q9 is "On" and Q5 is "Off". The charge injected into Q6 switches Q5 from Off to On, which attenuates the positive component of the signal and favors the negative component, producing a strong bipolar SET.

\section{F. Transients at Q6 in the NINV Configuration}

The NINV provides the weakest feed-back. The positive output signal is divided by a factor of 10 before being reinjected at the negative input terminal. Moreover, the quiescent voltage at this terminal is positive. As a result, the negative component of the SET is not strong enough to be visible on the output and no bipolar signal is observed. It is, however, strong enough to facilitate the circuit recovery; hence the sharp edges of the signal.

\section{G. Electrical Measurements}

The large-signal conditions that apply to most of the data lead, as already explained, to a straight-line recovery with a slope that becomes smaller with increasing TID. The large amount of energy deposited at a node in the circuit causes a large response on the output, which, in turn, is fed back to the input. Even in the INV and NINV cases, where the gain reduces the size of the feedback signal, the amplitude is still sufficiently large that the input transistors are driven into saturation. These are exactly the same conditions that pertain to the measurement of slew rate. In both cases the input signal is blocked from being amplified and the output does not follow the input. The result is a straight-line response. Therefore, measurement of the slew rate seems to be a good indicator of the slope of the recovery of the SET and its dependence on TID. For worst-case conditions, it is possible to estimate the duration of the longest SETs by measuring the slew rate and assuming the SET has maximum amplitude that equals the rail voltages. There was no obvious relationship between the degradation of the other electrical parameters, such as input offset voltage, and the shapes of the SETs.

\section{H. Total Ionizing Dose Effects}

An argument has been presented for a strong connection between the recovery of the SETs and the slew rate in the large-signal approximation. Fig. 12 shows that measurements of slew rate and recovery times are similar and that they degrade by the same amount following exposure to ionizing radiation.

The slew rate is determined by the re-charging of the compensating capacitor $\mathrm{C} 1$ by the current sources (Q15 and Q19). Exposure of a bipolar transistor to TID is known to result in a reduction in the transistor's gain, which, in turn, reduces the current drive capability of the current source [4]. Therefore, for large signal SETs, the change in the slope of the trailing edge with TID is directly attributable to the reduction in current drive of Q15 and Q19. This completely dominates the response of the circuit such that changes in the gains of the other transistors have no effect on the shapes of the SETs.

The data points for slew rate in Fig. 12 can be fit with a curve that is an exponentially decreasing function of TID, with a decay constant of $16 \mathrm{krad}(\mathrm{Si})$, i.e., that is the point where the slew rate has decreased to $1 / \mathrm{e}$ of its maximum value. (This fit is valid only over the TID range for this experiment and may not continue at higher doses.) The data points for open loop gain can also be fit with an exponential that has a very similar decay constant of $15 \mathrm{krad}(\mathrm{Si})$, suggesting that TID affects both parameters to the same degree.

Fig. 5 shows that the plateau voltage depends on TID. Fig. 15 is a plot of that voltage as a function of TID. The data may also be fit with an exponential curve having a decay constant of $17 \mathrm{krad}(\mathrm{Si})$, very close to those for slew rate and open loop gain. The change in plateau voltage may be attributed to changes in the turn-off voltages of the push-pull output stage (Q11 and Q13/Q14) induced by TID.

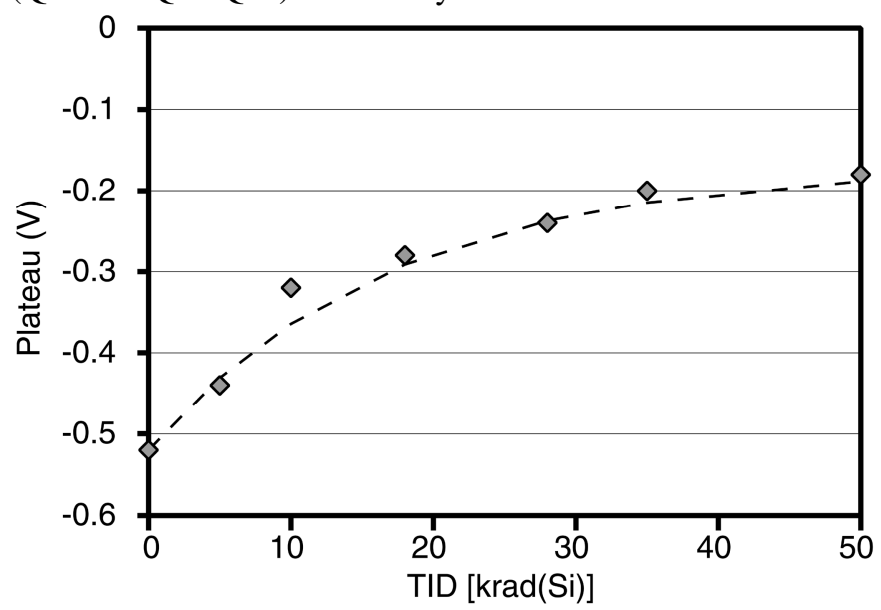

Fig. 15. Plateau voltage for the transistor Q9 in the NINV configuration as a function of TID.

\section{SUMMARY AND CONCLUSION}

We have shown that SETs in the LM124 operational amplifier have a variety of shapes and sizes, depending on 
where in the circuit they originate, on the amount of energy deposited, and on the electrical configuration of the amplifier. Data were taken for thirteen different nodes in three configurations, but because of space limitation, SETs for only three nodes were presented. Qualitative explanations for the shapes of the SETs originating at two transistors in three different configurations were presented. The recovery times for the large signal SETs were found to be comparable to the slew rates, and changes in recovery times and slew rates following TID exposure were found to be the same, leading to the conclusion that the changes are determined primarily by a reduction in the drive of the current sources as a result of TIDinduced reduction in transistor gain.

When it is necessary to use an unhardened linear bipolar part in a radiation environment where some of the electrical parameters will degrade, it may also be necessary to determine SET shape changes with TID. It is up to the design engineer to determine the type of SET mitigation to use, for which worst-case SET amplitudes and widths must be known. If it is not possible to measure SETs in irradiated parts using accelerator ions, the pulsed laser is an alternative. Alternatively, one can assume that the worst-case SETs reach the supply rail, which determines their amplitude, and the width is dominated by the recovery which may be estimated from the results of slew rate measurements.

\section{REFERENCES}

[1] M.F. Bernard, L. Dusseau, S. Buchner, D. McMorrow, R. Ecoffet, J. Boch, J.-R. Vaille, R.D. Schrimpf, and K. LaBel, "Impact of Total Ionizing Dose on the Analog Single Event Transient Sensitivity of a Linear Bipolar Integrated Circuit" IEEE Trans. Nucl. Sci. Vol. 54, pp. 2534 - 2540, Dec. 2007.

[2] S. Buchner, D. McMorrow, M.F. Bernard, N. Roche and L. Dusseau, "Total Dose Effect on Error Rates in Linear Bipolar Systems," accepted for publication in IEEE Trans. Nucl. Sci. Vol. 55, Aug. 2008.

[3] J. S. Melinger, S. Buchner, D. McMorrow, W. J. Stapor, T. R. Weatherford, and A. B. Campbell, "Critical evaluation of the pulsed laser method for single event effects testing and fundamental studies," IEEE Trans.Nucl. Sci., vol. 41, pp. 2574-2584, Dec. 1994.

[4] Y. Boulghassoul, L. W. Massengill, A.L. Sternberg, R. L. Pease, S. Buchner, J. W. Howard, D. McMorrow, M. W. Savage, and C. Poivey, "Circuit Modeling of the LM124 Operational Amplifier for Analog Single-Event Transient Analysis," IEEE Trans. Nucl. Sci., Vol. 49, No. 6, pp 3090-3096, Dec. 2002.

[5] P.R. Gray, P.J. Hurst, S.H. Lewis, and R.G. Meyer, "Analysis and Design of Analog Integrated Circuits,” New York: John Wiley \& Sons, Inc. 2001, ch. 5. 